\title{
Novel optical fiber sensor design for ground slope movement monitoring for railway safety operations
}

\author{
Daniel Hook, William Laing, Abdelfateh Kerrouche, Daniel Barreto and Lourdes Alwis \\ Edinburgh Napier University: School of Engineering and the Built Environment, Edinburgh EH10 5DT, UK
}

\begin{abstract}
Scour and slope instability are a major issue in keeping the railway structures safe and operational. Conventional measurement techniques do not allow real-time monitoring of slope movement and/or are time/resource consuming. Novel fiber optic technology could be utilized to continuously monitor variations in movement in the slopes along embankments adjacent to railway tracks so that early detection and warnings can be issued to avoid catastrophic events, as well as to enhance maintenance operations. To this end, a fiber optic Bragg grating-based sensor network embedded into a steel bar has been utilized to evaluate its feasibility to be installed in a railway embankment for slope movement monitoring. The paper highlights the initial laboratory results followed by a discussion on the practical implementation of the sensor system.
\end{abstract}

Keywords-Fibre Bragg Grating; scour monitoring; real-time sensing; slope movement monitoring; railway infrastructure safety

\section{INTRODUCTION}

Structural Health Monitoring (SHM) is a vital process within civil engineering and provides crucial data in analysing structure performance to ensure structural safety. Slope stability is seen to be a significant problem, where the main issue surrounds the full range of failure modes that can occur [1-3]. Scour and ground slope movement is one of the leading causes of bridge failure and at present in the UK alone, 4500 railway structures are at risk of scour, out of which 750 rated as high or medium/high risk [4]. Slope failure within the transport infrastructure cause undesirable economical, operational and environmental impacts, amongst others. Current monitoring techniques used for the purpose include laser scanning and Ground Penetrating Radar (GPR), tilt and inclinometer devices as well as standard surveying measurements [5-7]. However, equipment used for these techniques are bulky, often hard to assemble in some terrains, and require qualified personnel, as well as access and operation permissions within sensitive transport networks. This results in cost/time consumption. Additional comments could be made regarding the different levels of accuracy of these techniques, but such a discussion is out of the scope of this paper. Also, some of these techniques might not be appropriate during harsh environmental conditions, or those with difficult access. A key example is that of a flooding event causing scour and ground movements on adjacent structures. In such events, immediate assessment is required to ensure the safety of freight/passenger train operation on bridges. Suspension of traffic for inspection implies significant economical and reputational impact for train companies. Therefore, a measurement technique that can continuously monitor ground movements in real time and send out an alert if it is likely to cause damage to the bridge structure is warranted. The work presented herewith aims to tackle this issue utilizing smart Fiber optic sensors (FOS) based design that would monitor any ground movement which can be detected in real-time. FOS utilise glass fiber carrying light and possess a number of advantages such as being electrically passive (and hence safer to use), immune to electromagnetic interference, small in size (typically $<0.5 \mathrm{~mm}$ ), light weight and multiplexing capability, i.e. several sensors fabricated in a single fiber. In addition, optical fiber can handle a wider bandwidth of data which is ideal for systems involving vast amounts of sensing elements such as required to monitor the length of the embankment along railways lines.

Although optical fiber technology had been used thus far for slope/embankment strains and movements, the majority of monitoring had been limited to Brillouin Optical Time Domain Reflectometer (BOTDR) based techniques [8-10]. The objective of the work presented here is to explore the viability of Fibre Bragg Grating (FBG) based sensing mechanism to monitor slopes and embankments to act as an early failure warning system. Based on laboratory scale experiments, this work also includes a preliminary analysis of FOS performance on different types of soils to determine the consistency of failure patterns as well as the suitability of FOS for their measurement and interpretation.

\section{FABRICATION OF THE FUNCTIONALISED REINFORCING SENSOR SUBSTRATE}

\section{A. Fibre Bragg grating sensor fabrication}

The FBGs were fabricated using phase masks illuminated by light from a $248 \mathrm{~nm} \mathrm{KrF}$ excimer laser with a pulse energy of $12 \mathrm{~mJ}$ and a pulse frequency of $200 \mathrm{~Hz}$. The period of the phase masks used were carefully chosen so that their reflection wavelengths were within the C-band, to ensure the resonance wavelengths to be within the detection range of the interrogator. Micron Optics SM125 (with a capacity of simultaneous interrogation of approximately 40 sensors within the sensitivity range of the strain sensors fabricated for this work) was used for the purpose of interrogation. Following the fabrication, the gratings were annealed at $185^{\circ} \mathrm{C}$ for 3 hours to ensure temperature stability. The experiments were carried out at room temperature.

\section{B. Reinforcing substrate}

A series of $275 \times 10 \mathrm{~mm}$ diameter steel bars were manufactured with a $2 \mathrm{~mm}$ groove running along the grain of 
the bar to permit placement and protection of fibers and the sensors. Once the fiber had been placed within the groove, Araldite Instant Clear T epoxy was applied to encapsulate the fiber within the substrate as shown in Fig. 1.

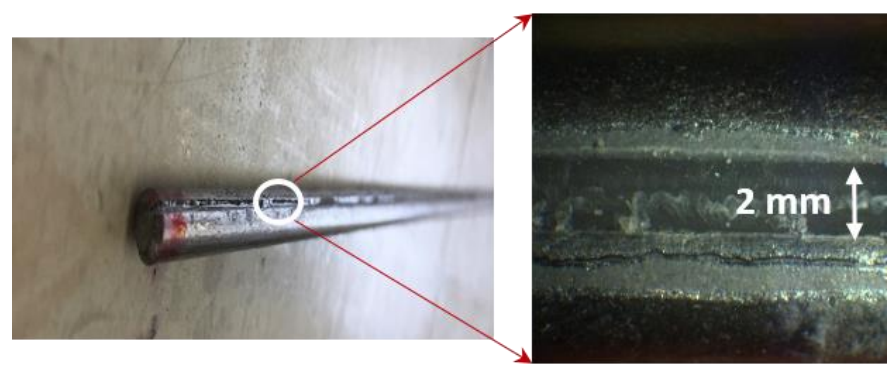

Fig. 1. Functionalised reinforcing sensor substrate (FRSS) with Araldite Instant Clear T epoxy coating.

The work presented here also evaluated the effect of different types of epoxy on strains perceived. Araldite Instant Clear $\mathrm{T}$ was found to be the most suitable in terms of consistency, protection and strain transfer.

\section{EXPERIMENTAL PROCEDURE}

The performance of the FRSS was evaluated through several tests and strain profiles were generated using Micron Optics Enlight software. Garside grade 14/25 sharp sand was used for the experiment and differing volumes of water were added to simulate different soil conditions. The soil that was tested had a moisture content of $2.5 \%$, which is a particular standard research sand used in geotechnical investigations. The choice moisture was based on preliminary tests to ensure test repeatability as well as an enhanced soil resistance due to the unsaturated soil condition generating water meniscii at inter-particle contacts, a well-recognized phenomenon well described in most soil mechanics textbooks.

The effects of temperature on strain measurements were also evaluated and it can be concluded that the effect would be negligible when considering large scale measurements and high load projects as the change in strain would have minimal effect on the identification of the initial slip zone. However, for smaller scale monitoring, this would need to be accounted for in an appropriate manner. Nevertheless, during the tests carried out in this work, the temperature was carefully monitored.

An experimental box was set up in order to simulate slope destabilization as shown in Fig. 2. Four FRSSs were inserted into the pre-made slope at pre-determined locations relative to the slip surface of the slope found by several initial destabilizations (as observed on the preliminary tests). One of the findings of the experiments here presented is that the orientation of the FRSS is crucial in obtaining accurate strain readings as shown in Fig. 3. In this instance, the FRSSs were placed to ensure that the groove was facing the ceiling (FRSS in compression).

The slope was destabilized by successfully subjecting 10 $\mathrm{kg}$ weights to a levelled off portion of the slope to eventually trigger a failure as seen in Fig. 4. Another testing method used was that of destabilizing the slope by using a gantry crane to tilt the box. However, due to the size of the box and the total load, this was not a practical option.

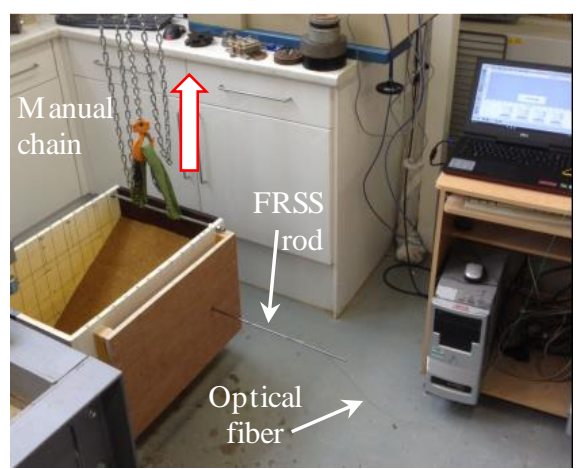

Fig. 2. Experimental box setup with bars inserted perpendicular to the plane.

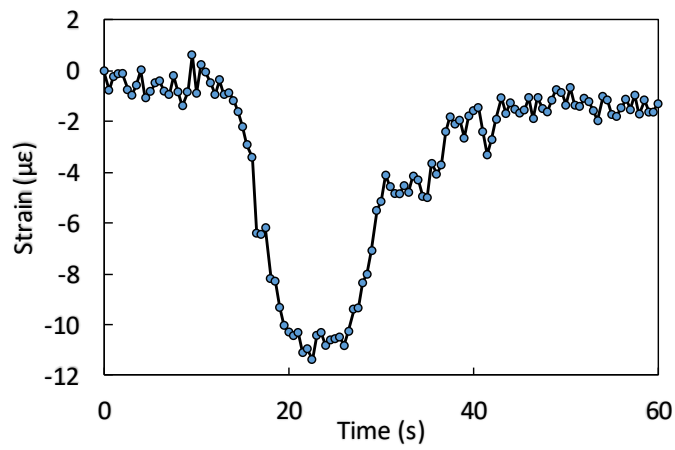

(a)

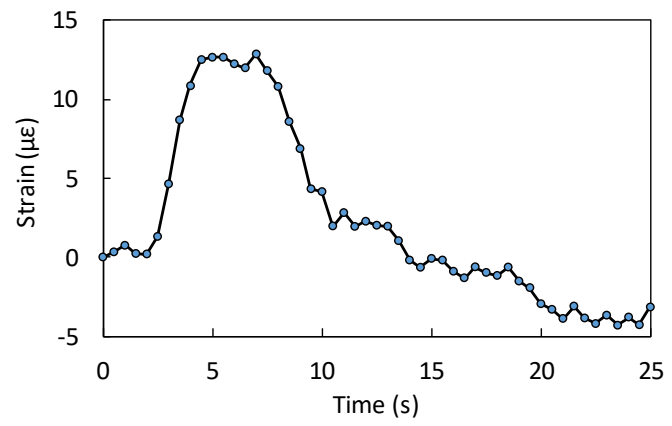

(b)

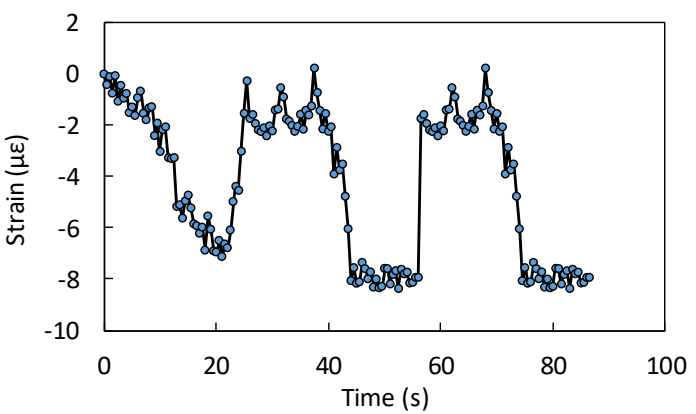

(c)

Fig. 3. Differences and irregularities in strain patterns of 3 FRSSs: (a) FRSS in compression, (b) FRSS in tension and (c) FRSS near axis of neutrality.

Data scanning commenced prior to loading to generate a datum point for the strain. Analysis of the data showed clear 
results in the strain perceived and the location of the initial failure was easily identifiable as illustrated in Fig. 5.

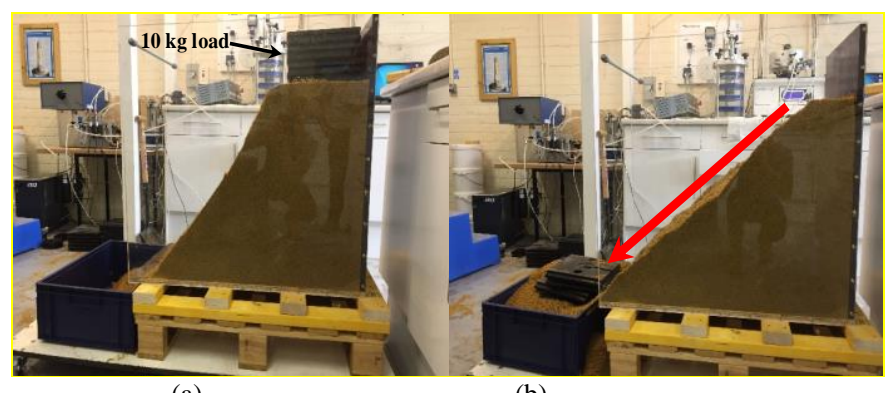

(a)

(b)

Fig. 4. Loading process: (a) loading and (b) failure of slope.

\section{RESULTS AND DISCUSSION}

The results show the build-up of strain in the slope before failure and highlight the key points to observe for early failure detection. Despite these results, there were disparities noticed between the different FRSSs placed at different locations. For example, it could be seen in Fig. 5 that the strain profiles are almost opposite. This could be due to the rotation of FRSSs during loading or during failure, a result of the failure mechanism within the soil. However, in both cases it is clear to see where the initial failure occurs. Therefore, it is sufficient for providing an early warning alert to signify that there could be a potential danger or failure. In any case, the disparities between sensors can be systematically investigated and related to various soil types, conditions and failure mechanisms. This warrants further research.

It is important to highlight that the use of optical fibers is not new for the monitoring of civil engineering infrastructure. For example, Janmonta et al. [10] measured high strains when fibers were placed on an existing tunnel, where fibers were attached using an Araldite epoxy resin. However, Janmonta's study was conducted to evaluate deformations of the tunnel instead of the ground. The works by Zhang et al. [9] describes the placement of FBGs near a potential slip surface in a centrifuge model of a slope. They found that FBGs are sensitive to temperature and realized that keeping ground temperature consistent in experiments was difficult. Although the work presented here is similar to that by Zhang et al. [9], it differs in terms of location and orientation of fibers, as well as the testing techniques. To the authors' best knowledge, the technique within the ground itself for slope monitoring as presented here is innovative.

In addition, as can be seen from Fig. 3, strain measurement of less than $10 \mu \varepsilon$ can be detected, which opens up the possibility of utilising the sensors for applications requiring sensitivities much higher than for the intended application here.

\section{CONCLUSION}

From a systematic set of laboratory scale experiments, it can be concluded that FBGs can be used for landslide detection and early warning. Considering the conditions simulated during the tests carried out in this study, the findings provide evidence that FBGs can be used as an initial failure detection system in slopes and embankments and are highly durable and flexible.

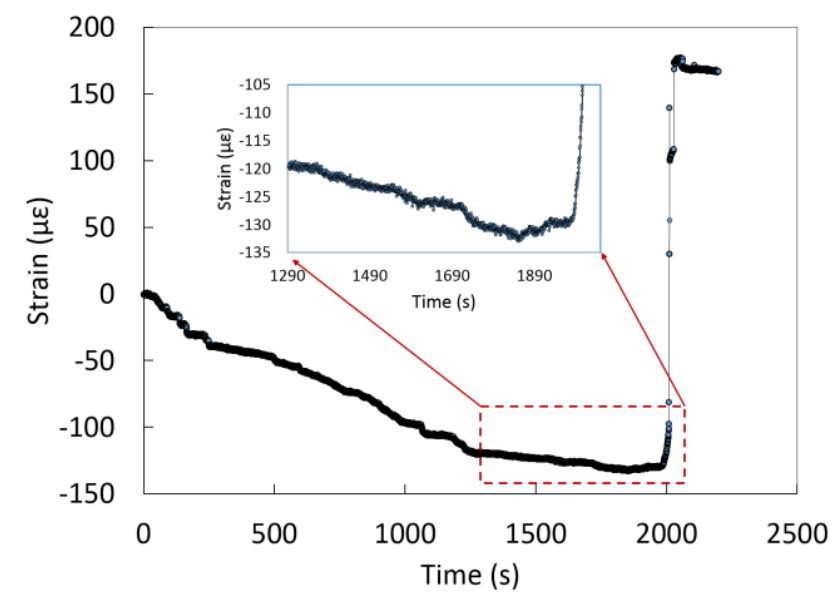

(a)

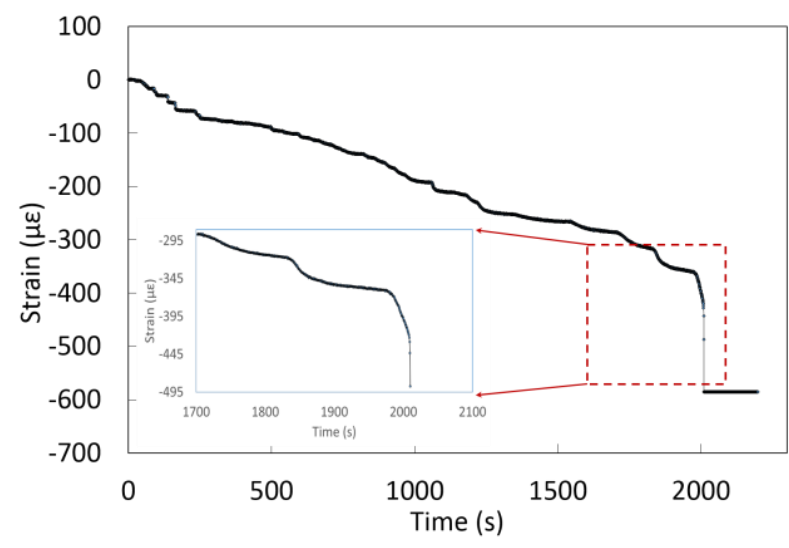

(b)

Fig. 5. Strain profiles of (a) FRSS (in compression) and (b) FRSS (in tension).

The study has also verified that precise locations of initial strain as well as strain build up can be identified in conjunction with early failure warnings. It was also observed that the location of the sensing element is of significant importance if interpretations of strain magnitude are to be made. The possibility of the detection of strain build-up is most valuable in sites where existing technologies would be difficult and even dangerous (cliffs, hard to access areas, railways etc.) to access. Even though these experiments were conducted over relatively short periods of time, they are still relatable to the field as progressive loading/destabilisation and deterioration of structures, where geotechnical features are inevitable.

Creep tests are currently carried out and scour tests investigated as in the field, slopes and embankments will be subjected to water level variation and saturation. Sensors are also being examined under harmonic periodic loading to determine if vibration affects the strain measurement. Further tests are currently being carried out to evaluate sensor response to extended lengths and their accuracy for detecting failure location, including tests at a railway embankment site. 


\section{REFERENCES}

[1] H. Li, D. Li, \& G. Song, "Recent applications of fibre optic sensors to health monitoring in civil engineering" in Engineering Structures (2004) Vol. 26 (11), pp. 1647-1657.

[2] Minardo et al., Soil slope monitoring by use of a Brillouin distributed sensor. IET Conference Proceedings 2015.

[3] S. E. Cho, "First-order reliability analysis of slope considering multiple failure modes" in Engineering Geology (2013).

[4] J. Briaud, P. Gardoni, C. Yao, "Statistical, risk, and reliability analyses of bridge course" in Journal of Geotechnical and Geoenvironmental Engineering (2014) Vol. 140, Iss.2.

[5] K. Osasan \& S. Afeni, "Review of surface mine slope monitoring techniques" in Journal of Mining Science (2010) Vol. 46(2), pp.177186.
[6] X. Meng, et al., "The Slope Monitoring Method Research" in Applied Mechanics and Materials (2014) Vol. 501-504, pp. 834-838.

[7] W. F. Kane and T. J. Beck, "Rapid slope monitoring in civil engineering," in American Society of Civil Engineer (1996) Vol. 666 New York.

[8] Damiano et al., "A Laboratory Study on the Use of Optical Fibers for Early Detection of Pre-Failure Slope Movements in Shallow Granular Soil Deposits" in Geotechnical Testing Journal (2017) Vol. 40(4), pp. 529-541.

[9] D. Zhang et al., "Internal deformation monitoring for centrifuge slope model with embedded FBG arrays" in Landslides (2017) Vol. 14(1), pp.407-417.

[10] K. Janmonta, T. Uchimura, B. Amatya, K. Soga, P. Bennett, R. Lung and I. Robertson, "Fibre Optics Monitoring of Clay Cuttings and Embankments along London's Ring Motorway", 2008, Cambridge: GEOCONGRESS. 